\title{
PERFECT RECONSTRUCTION SCHEMES FOR SAMPLING PIECEWISE SINUSOIDAL SIGNALS
}

\author{
Jesse Berent and Pier Luigi Dragotti \\ Electrical and Electronic Engineering \\ Imperial College London, Exhibition Road, London SW7-2AZ, UK \\ \{jesse.berent,p.dragotti\}@imperial.ac.uk
}

\begin{abstract}
Consider sampling a signal that is piecewise sinusoidal. Classical sampling theory does not enable a perfect reconstruction of the continuous time signal since the band is not limited [1]. However, we show that it is still possible to recover all the parameters of the sinusoids and the exact locations of the discontinuities using the annihilating filter method and recently developed Finite Rate of Innovation (FRI) sampling schemes [2,3]. Moreover, we show that there is a tradeoff between the number of sinusoids per piece and the proximity of the discontinuities in order to have a unique solution. This result recalls a sort of uncertainty principle.
\end{abstract}

\section{INTRODUCTION}

Back in 1949, Shannon formulated the famous sampling theorem [1]. It states that any bandlimited function $x(t)$ such that $X(\omega)=0 \forall$ $|\omega|>\omega_{\max }$ can be exactly recovered from its samples given that the rate $2 \pi / T$ is greater or equal to twice the highest frequency component. The continuous time signal is recovered with $x(t)=$ $\sum_{k \in \mathbb{Z}} y[k] \operatorname{sinc}(t / T-k)$ where $\operatorname{sinc}(t)=\sin (\pi t) / \pi t$ and $y[k]=$ $x(k T)$. It follows that any bandlimited signal can be expressed as a linear combination of an infinite length kernel. This sampling setup provides resolution in frequency however the infinite support of the sinc function causes the lack of resolution in time. Namely, an event concentrated in time cannot be precisely measured with this scheme.

It has recently been shown that certain classes of parametric signals that are not bandlimited can also be reconstructed from their samples. The notion on which these schemes rely is the rate of innovation defined in [2] as the counter for the number of degrees of freedom per unit of time. The authors show that signals with a finite rate of innovation (FRI) are uniquely determined from their samples given certain hypotheses. In particular, it is shown that streams of Diracs, differentiated Diracs and piecewise polynomials are recoverable. Furthermore, it has been shown in [3] that it is possible to recover exactly these signals using compact support kernels. As opposed to the sampling theorem, these results provide a setup that allows for precise time localization. But, to some extent, lacks frequency localization capabilities.

In this paper, we provide an approach for sampling signals with a combination of time and frequency components using local reconstruction schemes. More specifically, we consider piecewise sinusoidal functions. We use the annihilating filter method to recover the parameters of the sinusoids and FRI sampling schemes to recover the discontinuities. Furthermore, we put forward the tradeoff between time and frequency resolution which is in spirit similar to the uncertainty principle in $[4,5,6,7]$.
The paper is organized as follows: In Section 2 we define a piecewise sinusoidal signal and describe the sampling setup. Section 3 recalls the annihilating filters for exponentials and the results for sampling Diracs and differentiated Diracs. We also give a brief review of Exponential Splines (E-Splines) [8]. We then present a new sampling theorem in Section 4 for piecewise sinusoidal signals and conclude in Section 5.

\section{SIGNALS OF INTEREST AND SAMPLING SETUP}

The signals of interest in this paper are piecewise sinusoidal. Recall that a sinusoidal function can be expressed as the sum of two complex exponentials

$$
\begin{aligned}
x_{\omega}(t) & =\sum_{n=0}^{N_{d}-1} A_{n} \cos \left(\omega_{n} t+\phi_{n}\right) \\
& =\frac{1}{2} \sum_{n=0}^{N_{d}-1} A_{n}\left[e^{j\left(\omega_{n} t+\phi_{n}\right)}+e^{-j\left(\omega_{n} t+\phi_{n}\right)}\right],
\end{aligned}
$$

where $A_{n}>0$ and that it has a discrete and finite spectrum. Piecewise sinusoids are expressed here as a linear combinations of cosines multiplied by rectangle functions

$$
x(t)=\sum_{d \in \mathbb{Z}} \sum_{n=0}^{N_{d}-1} A_{d, n} \cos \left(\omega_{d, n} t+\phi_{d, n}\right) \Pi_{t_{d}, t_{d+1}},
$$

where $\Pi_{t_{d}, t_{d+1}}=u\left(t-t_{d}\right)-u\left(t-t_{d+1}\right)$ and $u(t)$ is the Heaviside step function. Clearly, the resulting spectrum is not bandlimited as the Fourier domain is made of the convolution between Diracs and sinc functions. Fig. 1(a) shows an example of a piecewise sinusoidal signal with two discontinuities and a maximum of two cosines per piece.

Assume our signal is pre-filtered with a kernel $\varphi(t)$ and sampled by an acquisition device. The observed discrete signal is characterized by

$$
y[k]=\int_{-\infty}^{\infty} x(t) \varphi(t / T-k) d t=\langle x(t), \varphi(t / T-k)\rangle,
$$

where $T$ is the sampling period. As $x(t)$ is not bandlimited, the sampling process causes aliasing. However, we will show that given certain conditions on $\varphi(t)$ and considering a certain tradeoff between the number of sinusoids and the proximity of the discontinuities, we can perfectly recover $x(t)$. 


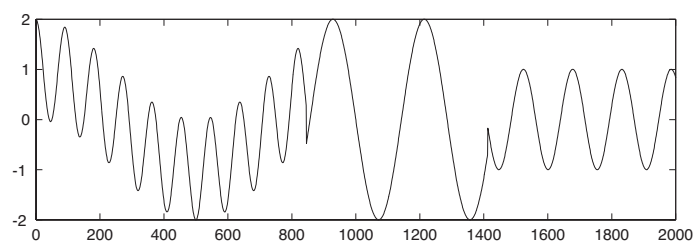

Fig. 1. Example of a piecewise sinusoidal signal with two discontinuities and a maximum of two sinusoids per piece.

\section{PRELIMINARIES}

In this section, we recall the annihilating filters for exponentials and the results shown in [3] for sampling streams of Diracs and differentiated Diracs. We also give a brief review of differential operators and cardinal E-Splines [8].

\subsection{Annihilating filters for exponentials}

A filter $h[k]$ is called annihilating filter of $s[k]$ if

$$
(h * s)[k]=0 \quad \forall k \in \mathbb{Z} .
$$

Assume $s[k]$ is made of a linear combination of weighted exponentials such that $s[k]=\sum_{n=0}^{N-1} a_{n} u_{n}^{k}$ with $u_{n} \in \mathbb{C}$. Clearly, the $(\mathrm{N}+1)$-tap filter with $z$-transform

$$
H_{\vec{u}}(z)=\prod_{n=0}^{N-1}\left(1-u_{n} z^{-1}\right)
$$

and $\vec{u}=\left(u_{0}, \ldots, u_{N-1}\right)$ will annihilate $s$. The knowledge of $H_{\vec{u}}(z)$ is sufficient to uniquely determine the $u_{n} \mathrm{~s}$. Indeed, (3) can be written in matrix-vector form with the Yule-Walker system $\mathbf{S} \cdot h_{\vec{u}}=0$ involving $2 \mathrm{~N}+1$ samples of $s$. Matrix $\mathbf{S}$ is therefore of size $N+1$ by $N+1$. The system has a unique solution as the annihilating filter is unique [9] and $\operatorname{Rank}(\mathbf{S})=N$. The weights $a_{n}$ are determined using $N+1$ samples to form a classic Vandermonde system which also has unique solution given that the $u_{n} \mathrm{~s}$ are distinct. Notice that a sinusoidal signal as defined in (1) can also be annihilated with a filter like $H_{\vec{u}}(z)$. Indeed, the filter is obtained by posing $\vec{u}=e^{\vec{\alpha}}$ and

$$
\vec{\alpha}=\left(j \omega_{0}, \ldots, j \omega_{N_{d}-1},-j \omega_{0}, \ldots,-j \omega_{N_{d}-1}\right) .
$$

We simplify the notation by expressing $H_{e^{\vec{\alpha}}}$ as $H_{\vec{\alpha}}$. Here $N=$ $2 N_{d}$. In practice, the annihilating filter is found using a singular value decomposition. We refer to [9] for a more detailed discussion.

\subsection{Sampling streams of differentiated Diracs}

For the sake of simplicity, we show the procedure for the basic case of sampling Diracs that are not differentiated and set the sampling period to $T=1$. The general case of reconstructing differentiated Diracs of maximum order $M-1$ is obtained using a similar procedure.

Let $x_{\delta}(t)$ be a stream of Diracs $x_{\delta}(t)=\sum_{n \in \mathbb{Z}} a_{n} \delta\left(t-t_{n}\right)$ sampled with a kernel $\varphi$ that can reproduce polynomials of maximum degree $N$. Therefore, there exists $c_{m, k}$ such that $\sum_{k} c_{m, k} \varphi(t-k)=$ $t^{m}$ for $m=0,1, \ldots, N$. The idea behind the necessity of the polynomial reproduction is that the weights and the locations are determined using the polynomial moments. Indeed, the annihilating filter method applied to $s[m]$ where

$$
\begin{aligned}
s[m] & =\sum_{k} c_{m, k} y[k] \\
& =\left\langle x(t), \sum_{k} c_{m, k} \varphi(t-k)\right\rangle \\
& =\int_{-\infty}^{\infty} x(t) t^{m} d t \\
& =\sum_{n=0}^{N-1} a_{n} t_{n}^{m}
\end{aligned}
$$

provides a unique solution for the $t_{n} \mathrm{~s}$ and $a_{n} \mathrm{~s}$ [3]. Equality (6) derives from the fact that $x_{\delta}(t)$ is a stream of Diracs and $\int f(t) \delta(t-$ $\left.t_{n}\right) d t=f\left(t_{n}\right)$. The general result is summarized as follows:

Theorem 1 (Dragotti, Vetterli, Blu [3]) Given is a sampling kernel $\varphi(t)$ that can reproduce polynomials of maximum degree $N \geq$ $2 K M-1$ and of compact support $L$. An infinite-length stream of Diracs $x(t)=\sum_{n=\mathbb{Z}} \sum_{m=0}^{M-1} a_{n, m} \delta^{(m)}\left(t-t_{n}\right)$ is uniquely determined by the samples $y_{n}=\langle\varphi(t / T-n), x(t)\rangle$ if and only if there are at most $K$ differentiated Diracs in an interval of length KLT.

\subsection{Differential operators and cardinal E-splines}

Consider a generic differential operator of order $N$

$$
L\{x(t)\}=\frac{d^{N} x(t)}{d t^{N}}+a_{N-1} \frac{d^{N-1} x(t)}{d t^{N-1}}+\ldots+a_{0} x(t)
$$

with constant coefficients $a_{n} \in \mathbb{C}$. This operator can also be defined by the roots of its characteristic polynomial $L(s)=\prod_{n=1}^{N}(s-$ $\left.\alpha_{n}\right)$. Using the same notation as in [8], we express the operator as $L_{\vec{\alpha}}$. Posing $s=j \omega$, we have in the frequency domain $L_{\vec{\alpha}}(j \omega)=$ $\prod_{n=1}^{N}\left(j \omega-\alpha_{n}\right)$. The null space of the operator, denoted $\mathcal{N}_{\vec{\alpha}}$, contains all the solutions to the differential equation $L\{x(t)\}=0$. As shown in [8], we have $\mathcal{N}_{\vec{\alpha}}=\operatorname{span}\left\{e^{\alpha_{n} t}\right\}_{n=1, \ldots, N}$. This is particularly interesting to us as a sinusoidal signal can be expressed as the linear combination of complex exponentials (1). Therefore, given that $\vec{\alpha}$ is as in (5), the operator $L_{\vec{\alpha}}$ will produce a zero output to the corresponding sinusoidal input. Put in other words, $x_{\omega}(t)$ is a solution to the differential equation $L_{\vec{\alpha}}\left\{x_{\omega}(t)\right\}=0$. This operator, together with the filter $H_{\vec{\alpha}}$, form the cardinal E-Splines introduced in [8]. These functions are extensions of the classical B-splines that are made of exponential segments instead of polynomial ones. Several interesting properties are derived in [8]. In particular, it is shown that a cardinal E-spline has finite support and has polynomial and exponential reproduction capabilities. The first order E-spline is a function $\beta_{\alpha}(t)$ with Fourier transform $\hat{\beta}_{\alpha}(\omega)=\frac{1-e^{\alpha-j \omega}}{j \omega-\alpha}$. The Esplines of order $N$ are constructed by $N$ successive convolutions of first order ones. In the Fourier domain we have [8]

$$
\hat{\beta}_{\vec{\alpha}}(\omega)=\prod_{n=0}^{N-1} \frac{1-e^{\alpha_{n}-j \omega}}{j \omega-\alpha_{n}}=\frac{H_{\vec{\alpha}}\left(e^{j \omega}\right)}{L_{\vec{\alpha}}(j \omega)} .
$$

It results that the annihilating filter for a linear combination of sinusoids can be expressed as the multiplication of a cardinal E-spline and a differential operator

$$
H_{\vec{\alpha}}\left(e^{j \omega}\right)=\hat{\beta}_{\vec{\alpha}}(\omega) L_{\vec{\alpha}}(j \omega) .
$$




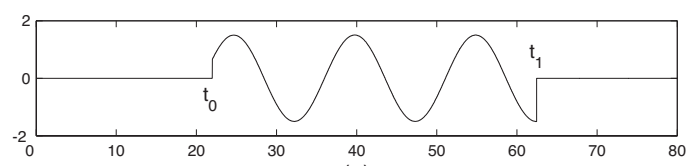

(a)

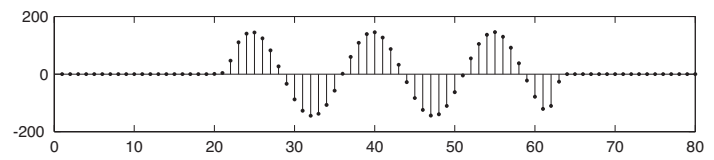

(b)

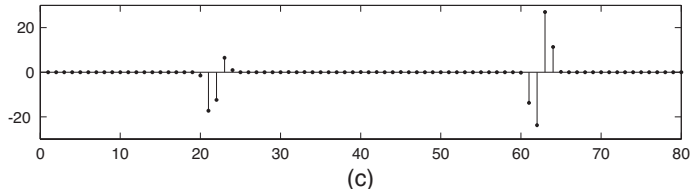

(c)

Fig. 2. (a) Original piecewise sinusoidal signal $x(t)$ with one sinusoid of frequency $\omega_{0}$. (b) Sampled signal $y[k]$ with kernel $\varphi(t)$. (c) Annihilated signal $y_{a n n}[k]=\left(y * h_{\vec{\alpha}}\right)[k]$ with $\vec{\alpha}=\left(j \omega_{0},-j \omega_{0}\right)$. These samples are equivalent to a sampled sum of differentiated Diracs in $t_{0}$ and $t_{1}$ with kernel $\varphi_{\text {equ }}(t)=\left(\varphi * \beta_{\vec{\alpha}}\right)(t)$.

\section{SAMPLING PIECEWISE SINUSOIDAL SIGNALS}

In this section, we consider recovering piecewise sinusoidal signals from their sampled versions. For clarity, we show the procedure with the most basic example and mention the results for the general case.

Let $x(t)$ be a sinusoid with frequency $\omega_{0}<\pi / T$ and truncated before $t_{0}$ and after $t_{1}$. Its continuous time representation is given by

$$
x(t)=A_{0} \cos \left(\omega_{0} t+\phi_{0}\right)\left[u\left(t-t_{0}\right)-u\left(t-t_{1}\right)\right],
$$

and the samples are given by (2). We use a filter of finite support $L$ and set the sampling period to $T=1$. Fig. 2(a) shows an example of such a signal and the observed samples are depicted in Fig. 2(b). There are two steps in the algorithm, namely the recovery of the sinusoids and the localization of the breakpoints. Both rely on the annihilating filter method.

\subsection{Recovering the sinusoids}

The first step in recovering the sinusoid is to find the samples that are not influenced by the discontinuities. Indeed, $4 N_{d}+1=5$ samples such that $k \in\left[t_{0}+L / 2, t_{1}-L / 2\right]$ where $L$ is the support of $\varphi$ are sufficient to determine $\omega_{0}$. A simple way to find these samples is through the rank of matrix $\mathbf{S}$. Recall from Section 3 that in this case $\mathbf{S}$ is a square matrix of size 3 by 3 and $\operatorname{Rank}(\mathbf{S})=2 N_{d}=2$. However, the matrix is full rank in the presence of a sample influenced by a breakpoint. Therefore, we slide a window along $k$ and run the annihilating filter method when the matrix is rank deficient. The filter in this case is $H_{\vec{\alpha}}$ where $\vec{\alpha}=\left(j \omega_{0},-j \omega_{0}\right)$. As shown in (4), $\omega_{0}$ is given by the roots of $H_{\vec{\alpha}}$. In order to find the amplitude and the phase, we use two consecutive samples to create the Vandermonde system

$$
\left[\begin{array}{cc}
e^{j \omega_{0} k} & e^{-j \omega_{0} k} \\
e^{j \omega_{0}(k+1)} & e^{-j \omega_{0}(k+1)}
\end{array}\right]\left[\begin{array}{c}
\tilde{A}_{0} e^{j \phi_{0}} \\
\tilde{A}_{0} e^{-j \phi_{0}}
\end{array}\right]=2\left[\begin{array}{c}
y[k] \\
y[k+1]
\end{array}\right]
$$

with $\tilde{A}_{0}=\hat{\varphi}\left(\omega_{0}\right) A_{0}$. The unicity of the solution is guarantied since the exponents are distinct. At this stage, all the parameters of the sinusoidal part are retrieved. The next step is to determine $t_{0}$ and $t_{1}$.

\subsection{Recovering the discontinuities}

The idea behind recovering the exact location of the breakpoints relies on Theorem 1. Indeed, if we apply the annihilating filter found in the previous step to the samples and develop the equation, we see that

$$
\begin{aligned}
y_{a n n}[k] & =\langle x(t), \varphi(t-k)\rangle * h_{\vec{\alpha}}[k] \\
& =\frac{1}{2 \pi}\left\langle X(\omega), e^{-j \omega k} \hat{\varphi}(\omega) H_{\vec{\alpha}}\left(e^{j \omega}\right)\right\rangle \\
& =\frac{1}{2 \pi}\left\langle X(\omega), e^{-j \omega k} \hat{\varphi}(\omega) \hat{\beta}_{\vec{\alpha}}(\omega) L_{\vec{\alpha}}(j \omega)\right\rangle \\
& =\frac{1}{2 \pi}\left\langle X(\omega) L_{\vec{\alpha}}(j \omega), e^{-j \omega k} \hat{\varphi}(\omega) \hat{\beta}_{\vec{\alpha}}(\omega)\right\rangle \\
& =\left\langle L_{\vec{\alpha}}\{x(t)\}, \varphi * \beta_{\vec{\alpha}}(t-k)\right\rangle,
\end{aligned}
$$

where we used Parseval's identity and (7). This means that the coefficients $y_{a n n}[k]$ shown in Fig. 2(c) represent the samples given by the inner-product between a modified $x(t)$ that we call $x_{\delta}(t)$ and a new kernel $\varphi_{\text {equ }}=\varphi * \beta_{\vec{\alpha}}$ with $\vec{\alpha}=\left(j \omega_{0},-j \omega_{0}\right)$. In the context of our example, we apply the differential operator and notice that

$$
\begin{aligned}
L_{\vec{\alpha}}\{x(t)\}= & \frac{d^{2} x(t)}{d t^{2}}+\omega_{0}^{2} x(t) \\
= & a_{0}(t)\left(\delta\left(t-t_{0}\right)-\delta\left(t-t_{1}\right)\right) \\
& +a_{1}(t)\left(\delta^{\prime}\left(t-t_{0}\right)-\delta^{\prime}\left(t-t_{1}\right)\right),
\end{aligned}
$$

with $a_{0}(t)=-2 A_{0} \omega_{0} \sin \left(\omega_{0} t+\phi_{0}\right)$ and $a_{1}(t)=A_{0} \cos \left(\omega_{0} t+\right.$ $\left.\phi_{0}\right)$. Therefore we conclude that sampling the signal and applying the annihilating filter is equivalent to sampling a stream of weighted and differentiated Diracs with the modified kernel $\varphi_{\text {equ }}$. In virtue of Theorem 1 we can find the exact locations of the discontinuities providing all the hypotheses are met. In this simple case, we only have differentiated Diracs of maximum order 1 and two locations $t_{0}$ and $t_{1}$. As we locate the $t_{n} \mathrm{~s}$ separately, we have $K=1$ and $M=2$. Therefore, according to Theorem 1 , we need a sampling kernel that can reproduce polynomials of degree $\geq 3$. Assume that $\varphi$ fulfills that condition. It is shown in [8] that the equivalent kernel $\varphi_{e q u}$ also fulfills the condition. As described in Section 3, we recover the locations $t_{0}$ and $t_{1}$ from $y_{a n n}$ by computing the roots of the annihilating filter for the polynomial moments $s[\mathrm{~m}]$ defined in (6). We refer to [3] for a detailed description.

\subsection{Generalization and discussion}

We have shown with this example how to recover all the parameters that characterize a truncated sinusoid. The general case is omitted in this paper due to lack of space. However, we bring attention to the fact that we use the annihilating filter $H_{\vec{\alpha}_{d}} \cdot H_{\vec{\alpha}_{d+1}}$ when there are two consecutive pieces with sinusoids. Here $\vec{\alpha}_{d}$ and $\vec{\alpha}_{d+1}$ contain the frequencies present in the first and the second pieces respectively. The resulting filter thus annihilates both sides of the discontinuity and the problem of finding the location is again reduced to that of finding differentiated Diracs. The complexity of the algorithm is polynomial and depends only on the number of sinusoids in two consecutive pieces. Furthermore, all the reconstruction schemes are local therefore the method can be extended to an infinite length piecewise sinusoidal signal. The following breakpoint is found using filter $H_{\vec{\alpha}_{d+1}} \cdot H_{\vec{\alpha}_{d+2}}$ and so on. The pseudocode for the algorithm and description of the procedure appears below: 
Find rank deficient windows

For each window

Find annihilating filter

Recover frequencies

Solve Vandermonde system

Recover amplitudes and phases

end for

For each pair of consecutive pieces

Create the composite annihilating filter

Filter the samples

end for

Recover discontinuity with Theorem 1

There are two main conditions to provide unique reconstruction. The first one is related to Theorem 1. Indeed, the equivalent signal $x_{\delta}(t)$ obtained after annihilation contains differentiated Diracs up to order $2 D-1$ where $D$ is the number of sinusoids in two consecutive pieces. Therefore, the sampling kernel $\varphi_{\text {equ }}(t)$ of support $L+2 D$ must be able to reproduce polynomials of degree $4 D-1$. The second condition is related to the length of the annihilating filter for sinusoids. Recall from Section 3 that we need $4 N_{d}+1$ samples to find the frequencies in each piece. From these bounds, we deduce the tradeoff between $N_{d}$, the number of sinusoids in the piece and $I_{d}=t_{d+1}-t_{d}$, the length of the piece. Indeed, the larger the $N_{d}$, the longer $I_{d}$ must be. Inversely, when $N_{d}$ decreases, $I_{d}$ can be shorter. There is a parametric uncertainty principle underlying that states $I_{d} /\left(4 N_{d}+L\right) \geq T$. We summarize the result in the following theorem:

Theorem 2 Given is a sampling kernel $\varphi(t)$ of compact support $L$ and that can reproduce polynomials of maximum degree $P \geq$ $4 D-1$. An infinite-length piecewise sinusoidal signal is uniquely determined by the samples $y_{n}=\langle\varphi(t / T-n), x(t)\rangle$ if there are at most $D$ sinusoids in two consecutive pieces and at most $N$ sinusoids with maximum frequency $\omega_{\max }<\pi / T$ in a piece of length $T(4 N+L)$.

A simulation for recovering a piecewise sinusoidal signal with two discontinuities and one sinusoid per piece is shown in Fig. 3. We use a classical B-Spline sampling kernel $\varphi(t)=\beta^{7}(t)$ as it is capable of reproducing polynomials of maximum degree $4 D-1=$ 7. The reconstructed signal is exact within machine precision.

\section{CONCLUSIONS}

We considered sampling piecewise sinusoidal signals and have shown that it is possible to recover exactly all the parameters that characterize it. Furthermore, we have put forward a parametric uncertainty principle that states that there is a tradeoff between the number of sinusoids in each piece and the proximity of the discontinuities.

We believe that a natural application of our result is in the spread spectrum communication systems. In particular in the case of frequency hopping signals, where precise frequency estimation and synchronization are of crucial importance. The design of a decoder for such signals that uses our sampling results and works at a rate below the rates normally used is under investigation.

\section{REFERENCES}

[1] C.E. Shannon, "Communications in the presence of noise," Proc. of the IRE, vol. 37, pp. 10-21, January 1949.

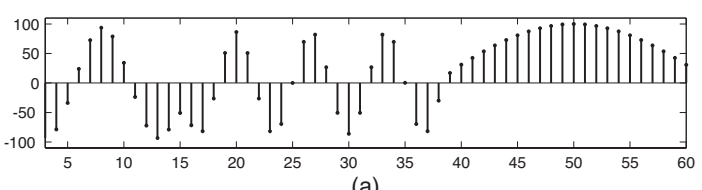

(a)

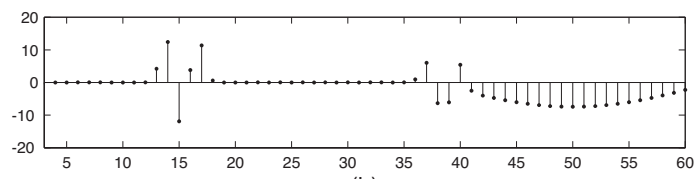

(b)

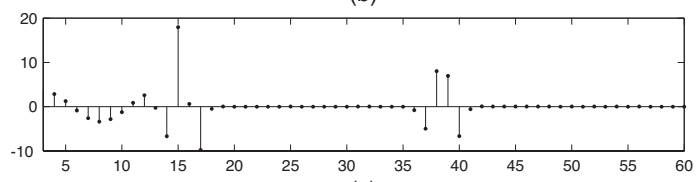

(c)

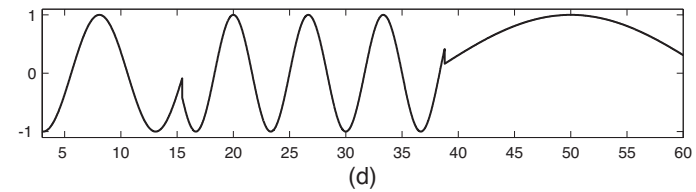

Fig. 3. Example of the reconstruction of a piecewise sinusoidal signal with one sine wave per piece and frequencies $\omega_{0}, \omega_{1}$ and $\omega_{2}$ in the first, second and third pieces respectively. (a) The observed samples. (b) The annihilated signal $y_{1}^{a n n}[k]=\left(y * h_{\vec{\alpha}_{0}} * h_{\vec{\alpha}_{1}}\right)[k]$ with $\vec{\alpha}_{0}=\left(j \omega_{0},-j \omega_{0}\right)$ and $\vec{\alpha}_{1}=\left(j \omega_{1},-j \omega_{1}\right)$. (c) The annihilated signal $y_{2}^{a n n}[k]=\left(y * h_{\vec{\alpha}_{1}} * h_{\vec{\alpha}_{2}}\right)[k]$ with $\vec{\alpha}_{1}=\left(j \omega_{1},-j \omega_{1}\right)$ and $\vec{\alpha}_{2}=\left(j \omega_{2},-j \omega_{2}\right)$. (d) The reconstructed continuous time signal.

[2] M. Vetterli, P. Marziliano, and T. Blu, "Sampling signals with finite rate of innovation," IEEE Trans. on Signal Processing, vol. 50, pp. 1417-1428, June 2002.

[3] P. L. Dragotti, M. Vetterli, and T. Blu, "Exact sampling results for signals with finite rate of innovation using the Strang-Fix conditions and local kernels," in Proc. of IEEE ICASSP, March 2005.

[4] D. Donoho and P. Stark, "Uncertainty principles and signal recovery," SIAM J. Appl. Math., vol. 49, no. 3, pp. 906-931, June 1989.

[5] D. Donoho and X. Huo, "Uncertainty principles and ideal atomic decompostion," IEEE Transactions on Information Theory, vol. 47, no. 7, pp. 2845-2862, November 2001.

[6] M. Elad and A. Bruckstein, "A generalized uncertainty principle and sparse representation in pairs of bases," IEEE Transactions on Information Theory, vol. 48, no. 9, pp. 2558-2567, September 2002.

[7] E. J. Candes, J. Romberg, and T. Tao, "Robust uncertainty principles: exact signal reconstruction from highly incomplete frequency information," Tech. Rep., California Institute of Technology, June 2004, submitted to IEEE Trans. on Information Theory.

[8] M. Unser and T. Blu, "Cardinal exponential splines: Part ITheory and filtering algorithms," IEEE Trans. on Signal Processing, vol. 53, no. 4, pp. 1425-1438, April 2005.

[9] P. Stoica and R. Moses, Introduction to Spectral Analysis, Prentice Hall, Englewood Cliffs, NJ, 2000. 